\title{
THE CORE LABEL ORDER OF A CONGRUENCE-UNIFORM LATTICE
}

\author{
HENRI MÜHLE
}

\begin{abstract}
We investigate the alternate order on a congruence-uniform lattice $\mathcal{L}$ as introduced by N. Reading, which we dub the core label order of $\mathcal{L}$. When $\mathcal{L}$ can be realized as a poset of regions of a simplicial hyperplane arrangement, the core label order is always a lattice. For general $\mathcal{L}$, however, this fails. We provide an equivalent characterization for the core label order to be a lattice. As a consequence we show that the property of the core label order being a lattice is inherited to lattice quotients. We use the core label order to characterize the congruence-uniform lattices that are Boolean lattices, and we investigate the connection between congruence-uniform lattices whose core label orders are lattices and congruence-uniform lattices of biclosed sets.
\end{abstract}

\section{INTRODUCTION}

A (real) hyperplane arrangement $\mathcal{A}$ is a collection of hyperplanes in $\mathbb{R}^{n}$, and the connected components of $\mathbb{R}^{n} \backslash \mathcal{A}$ are called the regions of $\mathcal{A}$. P. Edelman defined a partial order on the set of regions of $\mathcal{A}$ with respect to a fixed base region: two regions are comparable in this order whenever we can go from the one region to the other by crossing one hyperplane at a time and never decreasing the number of hyperplanes between the current region and the base region [8].

It was shown in [4] that this poset of regions is a lattice whenever $\mathcal{A}$ is simplicial. Subsequently, N. Reading thoroughly studied the structure of the poset of regions [20,21], see also [26]. One of the main results in his study is a characterization of those hyperplane arrangements that have posets of regions which are semidistributive or congruence-uniform lattices, see Theorem 9-3.8 and Corollary 9-7.22 in [26]. See also [15, Theorem 3].

A key tool for understanding lattice congruences in the lattice of regions (and therefore congruence-uniformity) are so-called shards of hyperplanes. The terminology suggests that these can be understood as pieces of hyperplanes that are broken off by intersections with other (in some sense stronger) hyperplanes. Proposition 3.3 in [24] states that the shards of a simplicial hyperplane arrangement $\mathcal{A}$ are in bijection with the join-irreducible elements of the lattice of regions (and thus, if this lattice is congruence uniform, with the join-irreducible lattice congruences). The shards give rise to an alternate partial order on the regions of $\mathcal{A}$ : the shard intersection order. It turns out that this order is always a lattice [24, Section 4]. Perhaps the most prominent example of a shard intersection order is the lattice of noncrossing partitions associated with a finite Coxeter group, which arises from

2010 Mathematics Subject Classification. 06B05 (primary), and 06A07 (secondary).

Key words and phrases. congruence-uniform lattices, interval doubling, semidistributive lattices, crosscut theorem, Möbius function, biclosed sets. 
certain quotient lattices of the poset of regions of the corresponding Coxeter arrangement [24, Theorem 8.5]. These quotient lattices are known as Cambrian lattices; see $[22,23]$ for more background. The shard intersection order of the lattice of regions of a Coxeter arrangement was also studied in [1,2,19].

$\mathrm{N}$. Reading suggested a generalization of the shard intersection order to arbitrary congruence-uniform lattices [26, Section 9-7.4], where he essentially associates a certain set of join-irreducible elements with each lattice element, and then orders these sets by containment. This article is devoted to the study of this order, which we decided to call the core label order; denoted by $\operatorname{CLO}(\mathcal{L})$. The terminology is due to the fact that $\mathrm{CLO}(\mathcal{L})$ can be realized as a family of sets of certain edgelabels in the poset diagram of $\mathcal{L}$ ordered by inclusion. See Section 3.1 for further justification of this terminology.

It turns out that at this level of generality the lattice property of the core label order is no longer guaranteed. Problem 9.5 in [26] asks for conditions on $\mathcal{L}$ such that $\operatorname{CLO}(\mathcal{L})$ is again a lattice. The first main result of this article is a necessary condition stating that if $\operatorname{CLO}(\mathcal{L})$ is a lattice, then $\mathcal{L}$ is spherical, i.e. the order complex of the proper part of $\mathcal{L}$ is homotopic to a sphere.

Theorem 1.1. Let $\mathcal{L}$ be a congruence-uniform lattice. If $\operatorname{CLO}(\mathcal{L})$ is a lattice, then $\mathcal{L}$ is spherical.

The proof of Theorem 1.1 essentially follows from the semidistributivity of $\mathcal{L}$ and G.-C. Rota's Crosscut Theorem. This condition is, however, not sufficient if $\mathcal{L}$ has more than eight elements. We can explicitly construct spherical congruenceuniform lattices with at least nine elements whose core label order is not a lattice.

Theorem 1.2. For all $n \geq 9$ there exists a spherical congruence-uniform lattice of cardinality $n$ whose core label order is not a lattice.

The smallest example of the family of congruence-uniform lattices that occur in Theorem 1.2 is the Boolean lattice of size eight doubled by an atom. We prove Theorems 1.1 and 1.2 in Section 4.1, after we have recalled the necessary latticetheoretic notions in Section 2, and have laid some more groundwork in Section 3.1.

If $\mathcal{L}$ is spherical, then an easy sufficient condition for the lattice property of $\operatorname{CLO}(\mathcal{L})$ is that the family of core label sets of $\mathcal{L}$ is closed under intersection. If this is satisfied, we say that $\mathcal{L}$ has the intersection property, and we show that this property is in fact equivalent to $\operatorname{CLO}(\mathcal{L})$ being a meet-semilattice. We may conclude the following result.

Theorem 1.3. The core label order of a congruence-uniform lattice $\mathcal{L}$ is a lattice if and only if $\mathcal{L}$ is spherical and has the intersection property.

Of course, we may now ask for conditions on $\mathcal{L}$ that ensure the intersection property. We are able to show that the intersection property is inherited to lattice quotients, which in view of Theorem 1.3 implies that the class of congruenceuniform lattices whose core label orders are lattices is closed under taking quotients.

Theorem 1.4. Let $\mathcal{L}$ be a spherical congruence-uniform lattice with the intersection property. For any lattice congruence $\Theta$ of $\mathcal{L}$, the core label order of the quotient lattice $\mathcal{L} / \Theta$ is a lattice. 
The proofs of Theorems 1.3 and 1.4 are both given in Section 4.2, where we also formally define the intersection property.

In Section 5 we show that $\mathcal{L}$ and $\operatorname{CLO}(\mathcal{L})$ intersect in a common Boolean lattice, which we call the Boolean nexus of $\mathcal{L}$. This lattice is closely related to the crosscut complex of $\mathcal{L}$. We also show that the Boolean lattices are the only congruenceuniform lattices that are atomic (Theorem 2.10), i.e. in which every element can be written as a join of atoms. This observation enables us to prove the following result, which is a new characterization of Boolean lattices in terms of the core label order.

Theorem 1.5. Let $\mathcal{L}$ be a congruence-uniform lattice. We have $\mathcal{L} \cong \mathrm{CLO}(\mathcal{L})$ if and only if $\mathcal{L}$ is a Boolean lattice.

If we browse the current literature, then it seems that all the available congruenceuniform lattices whose core label orders are lattices have one thing in common: they are (quotients of) lattices of biclosed sets. Let us postpone the definition until Section 6.

For instance, Theorem 4.2.2 in [14] states that every lattice of regions of a simplicial hyperplane arrangement is a lattice of biclosed sets. Moreover, A. Garver and T. McConville define in [12] a lattice of biclosed sets of segments of a tree embedded in a disk, and they prove that it is congruence uniform. Theorems 5.12 and 5.14 in [12] imply that the core label order of certain quotients of this lattice has the lattice property; Theorem 5.13 in [5] states that the core label order of the full lattice has the lattice property, too. In a similar spirit, T. McConville defines in [16] a lattice of biclosed sets of segments on a grid, and he proves that it is congruence uniform. Proposition 5.20 in [11] implies that the core label order of certain quotients of this lattice has the lattice property.

In Section 6 we explore the connection between congruence-uniform lattices whose core label orders have the lattice property and congruence-uniform lattices of biclosed sets. We exhibit a spherical congruence-uniform lattice of biclosed sets whose core label order is not a lattice. Moreover, in Problem 6.2 we ask for a graded spherical congruence-uniform lattice of biclosed sets whose core label order is not a lattice.

\section{BACKGROUND}

2.1. Lattices and Congruences. Let $\mathcal{L}=(L, \leq)$ be a lattice, i.e. a partially ordered set (poset for short) in which every two elements $x, y \in L$ have a greatest lower bound (their meet; written $x \wedge y$ ), and a least upper bound (their join; written $x \vee y$ ). Throughout the paper we will only consider finite lattices. It follows that $\mathcal{L}$ has a least element $\hat{0}$ and a greatest element $\hat{1}$.

Two elements $x, y \in L$ form a cover relation in $\mathcal{L}$ if $x<y$ and there is no $z \in L$ with $x<z<y$. We usually write $x \lessdot y$, and say that $x$ is a lower cover of $y$; or equivalently that $y$ is an upper cover of $x$.

The dual of $\mathcal{L}$ is the lattice $\mathcal{L}^{*} \stackrel{\text { def }}{=}(L, \geq)$. If $\mathcal{L} \cong \mathcal{L}^{*}$, then $\mathcal{L}$ is self dual.

An element $j \in L \backslash\{\hat{0}\}$ is join irreducible if whenever $j=x \vee y$ for $x, y \in L$, then $j \in\{x, y\}$. Since $\mathcal{L}$ is finite, it follows that every join-irreducible element $j$ has a unique lower cover $j_{*}$. Dually, we define the set of meet-irreducible elements of $\mathcal{L}$ by $\mathcal{M}(\mathcal{L}) \stackrel{\text { def }}{=} \mathcal{J}\left(\mathcal{L}^{*}\right)$. 


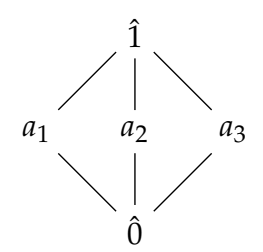

(a) A five-element lattice.

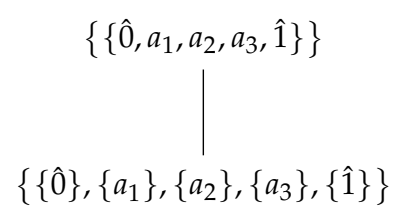

(b) The congruence lattice of the lattice in Figure 1a.

Figure 1. A lattice that is not congruence uniform.

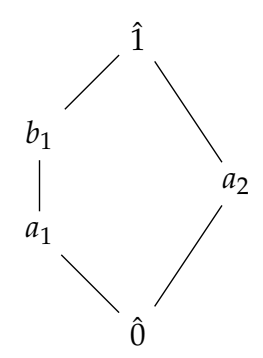

(a) Another five-element lattice.

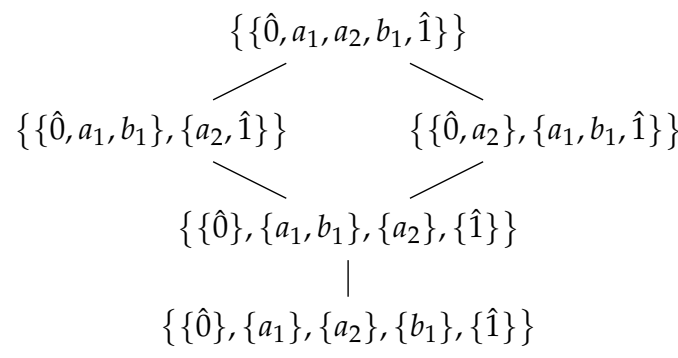

(b) The congruence lattice of the lattice in Figure 2a.

Figure 2. A congruence-uniform lattice.

A lattice congruence is an equivalence relation $\Theta$ on $L$ such that $[x]_{\Theta}=[y]_{\Theta}$ and $[u]_{\Theta}=[v]_{\Theta}$ imply $[x \wedge u]_{\Theta}=[y \wedge v]_{\Theta}$ and $[x \vee u]_{\Theta}=[y \vee v]_{\Theta}$ for all $x, y, u, v \in L$. The set $\operatorname{Con}(\mathcal{L})$ of all lattice congruences of $\mathcal{L}$ ordered by refinement is again a lattice [10]; the congruence lattice of $\mathcal{L}$. For $x, y \in L$ with $x \lessdot y$, let $\operatorname{cg}(x, y)$ denote the finest lattice congruence of $\mathcal{L}$ in which $x$ and $y$ are equivalent. If $y \in \mathcal{J}(\mathcal{L})$, then we write $\operatorname{cg}(y)$ instead of $\operatorname{cg}\left(y_{*}, y\right)$.

We have the following characterization of join-irreducible lattice congruences; see [13, Section 2.14] for the equivalence of (i) and (ii) and [9, Theorem 3.20] for the equivalence of (i) and (iii).

Theorem 2.1. Let $\mathcal{L}$ be a finite lattice, and let $\Theta \in \operatorname{Con}(\mathcal{L})$. The following are equivalent.

(i) $\Theta$ is join-irreducible in $\operatorname{Con}(\mathcal{L})$.

(ii) $\Theta=\operatorname{cg}(x, y)$ for some $x \lessdot y$.

(iii) $\Theta=\operatorname{cg}(j)$ for some $j \in \mathcal{J}(\mathcal{L})$.

The map $j \mapsto \operatorname{cg}(j)$ is surjective by Theorem 2.1, but in general it may fail to be injective. A finite lattice is congruence uniform if this map is a bijection for both $\mathcal{L}$ and $\mathcal{L}^{*}$.

Congruence-uniform lattices sometimes appear in the (universal algebra and lattice theory) literature under the name "bounded lattices", which has its origins in [17] and refers to the fact that these lattices are precisely the boundedhomomorphic images of a free lattice. This notation, however, clashes with the term "bounded poset", which refers simply to the fact that a poset has a least and a greatest element, and is widely used in combinatorics. 

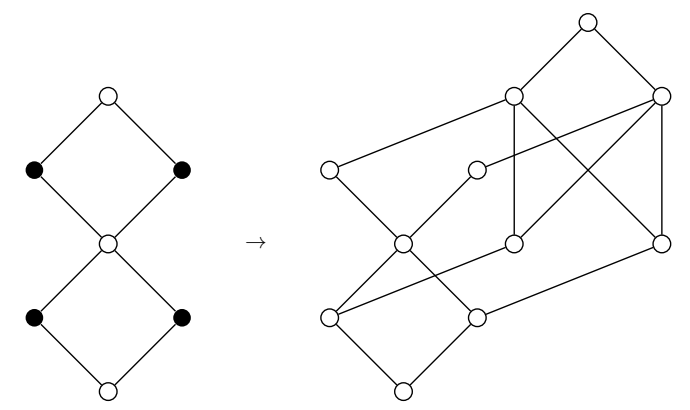

Figure 3. If we double the left lattice by the set of solid dots, then we obtain a non-lattice.

Figures 1 and 2 show two lattices with five elements together with their congruence lattices. The first example is not congruence uniform, the second one is.

For later use, let us record that the class of congruence-uniform lattices is a pseudovariety.

Proposition 2.2 ([6, Theorem 4.3]). Congruence-uniformity is preserved under taking quotients, sublattices and finite direct products.

2.2. Doubling by Intervals. It follows from a result of A. Day that congruenceuniform lattices can be characterized by means of the following doubling construction.

Let $\mathcal{P}=(P, \leq)$ be an arbitrary (finite) partially ordered set, and let $I \subseteq P$. Define $P_{\leq I} \stackrel{\text { def }}{=}\{x \in P \mid x \leq y$ for some $y \in I\}$. Let 2 denote the 2-element lattice on the ground set $\{0,1\}$, where we set $0<1$. The doubling of $\mathcal{P}$ by $I$ is the subposet of the direct product $\mathcal{P} \times \mathbf{2}$ given by the ground set

$$
\left(P_{\leq I} \times\{0\}\right) \uplus\left(\left(\left(P \backslash P_{\leq I}\right) \cup I\right) \times\{1\}\right),
$$

where " $\uplus$ " denotes disjoint set union. We denote the resulting poset by $\mathcal{P}[I]$, and if $I=\{i\}$ we write $\mathcal{P}[i]$ instead of $\mathcal{P}[\{i\}]$.

If $\mathcal{L}=(L, \leq)$ is a lattice, and $I \subseteq L$ is an arbitrary subset, then it is not necessarily true that $\mathcal{L}[I]$ is still a lattice. This is illustrated in Figure 3. If we restrict ourselves to doublings by order convex subsets, however, then it follows from [7] that the lattice property is preserved. (Recall that $I \subseteq P$ is order convex if for all $x, y, z \in P$ with $x<y<z$ we have that $x, z \in I$ implies $y \in I$.)

Lemma 2.3. Let $\mathcal{L}=(L, \leq)$ be a finite lattice, and let $I \subseteq L$ be order convex. If the induced subposet $(I, \leq)$ has exactly $k$ minimal elements, then

$$
|\mathcal{J}(\mathcal{L}[I])|=|\mathcal{J}(\mathcal{L})|+k
$$

Proof. Since $I$ is order convex it follows from [7] that $\mathcal{L}[I]$ is again a lattice. Let $x \in L$. We count the lower covers of $(x, 0)$ resp. $(x, 1)$ in $\mathcal{L}[I]$.

If $x \in L_{\leq I}$, then $(y, a)$ is a lower cover of $(x, 0)$ in $\mathcal{L}[I]$ if and only if $a=0$ and $y$ is a lower cover of $x$ in $\mathcal{L}$.

Now let $x \in L \backslash L_{\leq I}$, and suppose that $(y, a)$ is a lower cover of $(x, 1)$ in $\mathcal{L}[I]$. If $a=0$, then $y \in L_{\leq I} \backslash I$, and $y$ must be a lower cover of $x$ in $\mathcal{L}$. (If $y \in I$ is a lower 


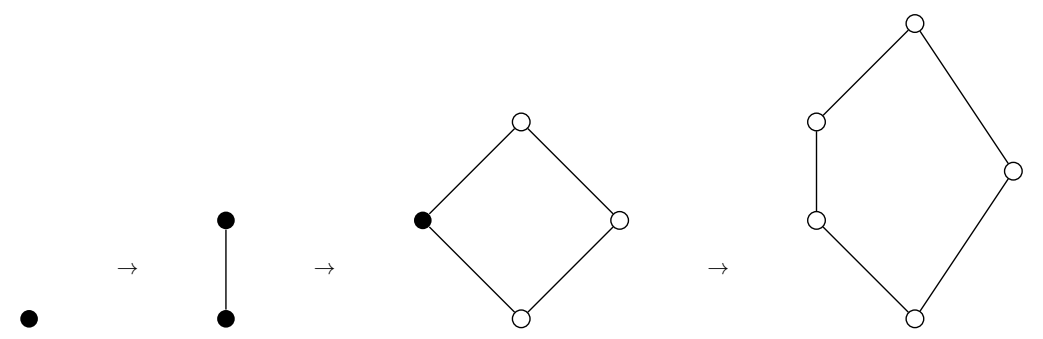

Figure 4. The lattice in Figure 2a can be obtained by a sequence of doublings.

cover of $x$ in $\mathcal{L}$ and $a=0$, then we have $(y, 0)<(y, 1)<(x, 1)$ in $\mathcal{L}[I]$, which is a contradiction.) If $a=1$, then $y \in\left(L \backslash L_{\leq I}\right) \cup I$, and $y$ must be a lower cover of $x$ in $\mathcal{L}$.

Finally, let $x \in I$, and suppose that $y_{1}, y_{2}, \ldots, y_{s}$ are the lower covers of $x$ in the induced subposet $(I, \leq)$. Then, the lower covers of $(x, 1)$ in $\mathcal{L}[I]$ are precisely $\left(y_{1}, 1\right),\left(y_{2}, 1\right), \ldots,\left(y_{s}, 1\right)$ and $(x, 0)$.

The doubling construction enables us to characterize congruence-uniform lattices in a second way.

Theorem 2.4 ([6, Theorem 5.1]). A finite lattice is congruence uniform if and only if it can be obtained from the singleton lattice by a sequence of doublings by intervals.

In particular, Lemma 2.3 implies that for a congruence-uniform lattice $\mathcal{L}$ the size of $\mathcal{J}(\mathcal{L})$ determines the exact number of doubling steps needed to create $\mathcal{L}$.

Figure 4 shows how the lattice in Figure 2a can be obtained by a sequence of three doublings. The intervals at which we double are marked by solid dots.

An important family of congruence-uniform lattices are the Boolean lattices $\operatorname{Bool}(M) \stackrel{\text { def }}{=}$ $(\wp(M), \subseteq)$, where $\wp(M)$ denotes the power set of $M$. If $M=[n] \stackrel{\text { def }}{=}\{1,2, \ldots, n\}$, then we usually write $\operatorname{Bool}(n)$ instead of $\operatorname{Bool}([n])$. Observe that we may obtain $\operatorname{Bool}(n)$ from the singleton lattice by exactly $n$ doublings, where we double at each step by the full lattice.

2.3. Semidistributive Lattices. A finite lattice $\mathcal{L}=(L, \leq)$ is join semidistributive if for every $x, y, z \in L$ with $x \vee y=x \vee z$ we have $x \vee(y \wedge z)=x \vee y$. We call $\mathcal{L}$ meet semidistributive if $\mathcal{L}^{*}$ is join semidistributive, and we say that $\mathcal{L}$ is semidistributive if it is both join and meet semidistributive.

Proposition 2.5 ([6, Lemma 4.2]). Every congruence-uniform lattice is semidistributive.

The converse implication is not true, as is witnessed for instance by the example in Figure 5. See also [18, Section 3]. Moreover, [9, Lemma 2.62] characterizes the semidistributive, congruence-uniform lattices.

Join-semidistributive lattices have the following characterizing property. A set $X \subseteq L$ is a join representation of $x \in L$ if $\bigvee X=x$. A join representation $X$ of $x$ is irredundant if there is no $X^{\prime} \subsetneq X$ with $x=\vee X^{\prime}$. If $X$ and $X^{\prime}$ are two irredundant join representations of $x$, then $X$ refines $X^{\prime}$ if for every $z \in X$ there exists $z^{\prime} \in X^{\prime}$ with $z \leq z^{\prime}$. A join representation of $x$ is canonical if it is irredundant and refines 


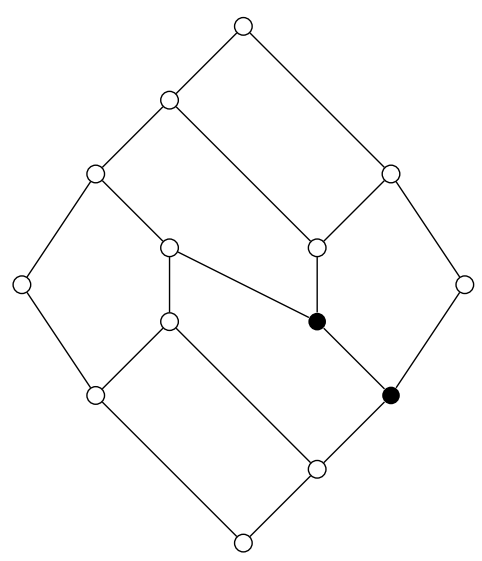

Figure 5. The smallest semidistributive lattice that is not congruence uniform. The two highlighted join-irreducible elements induce the same lattice congruence.

every other irredundant join representation of $x$; let us write $\Gamma(x)$ for the canonical join representation of $x$ (if it exists). We define (canonical) meet representations dually.

Theorem 2.6 ([9, Theorem 2.24]). A finite lattice is join semidistributive if and only if every element has a canonical join representation.

The next result states that the canonical join representations in fact form a simplical complex; see also [3].

Proposition 2.7 ([25, Proposition 2.2]). Let $(L, \leq)$ be a finite lattice, and let $X \subseteq L$. If $\bigvee X$ is a canonical join representation, and $X^{\prime} \subseteq X$, then $\bigvee X^{\prime}$ is also a canonical join representation.

Now suppose that $\mathcal{L}$ is congruence uniform, and pick $x, y \in L$ with $x \lessdot y$. Theorem 2.1 and the fact that $j \mapsto \operatorname{cg}(j)$ is a bijection imply that there is a unique $j \in \mathcal{J}(\mathcal{L})$ with $\operatorname{cg}(j)=\operatorname{cg}(x, y)$; we usually write $j_{\operatorname{cg}(x, y)}$ to denote this element. This property enables us describe canonical join representations in $\mathcal{L}$ explicitly.

Proposition 2.8 ([12, Proposition 2.9]). Let $\mathcal{L}=(L, \leq)$ be a congruence uniform lattice. The canonical join representation of $x \in L$ is

$$
\Gamma(x)=\left\{j_{\mathrm{cg}(y, x)} \mid y \lessdot x\right\} .
$$

An atom of $\mathcal{L}$ is an element $a \in L$ with $0 \lessdot a$. Let us write $\operatorname{Atoms}(\mathcal{L})$ for the set of atoms of $\mathcal{L}$. A coatom of $\mathcal{L}$ is an atom of $\mathcal{L}^{*}$.

Proposition 2.9. Let $\mathcal{L}$ be a meet semidistributive lattice, and let $X \subseteq \operatorname{Atoms}(\mathcal{L})$. If $\bigvee X=\hat{1}$, then $X=\operatorname{Atoms}(\mathcal{L})$.

Proof. Let $X \subsetneq \operatorname{Atoms}(\mathcal{L})$ with $\bigvee X=\hat{1}$, and let $a \in \operatorname{Atoms}(\mathcal{L}) \backslash X$. For every $x \in X$ we have $a \wedge x=\hat{0}$, since $a$ and $x$ are atoms. The meet-semidistributivity of $\mathcal{L}$ then implies that $\hat{0}=a \wedge(\bigvee X)=a \wedge \hat{1}=a$. This contradicts the assumption that $a$ is an atom, and we conclude $X=\operatorname{Atoms}(\mathcal{L})$. 
A lattice is atomic if every element can be expressed as a join of atoms. We conclude this section with the observation that Boolean lattices are the only atomic semidistributive lattices.

Theorem 2.10. Let $\mathcal{L}$ be a semidistributive lattice. Then, $\mathcal{L}$ is atomic if and only if $\mathcal{L} \cong \operatorname{Bool}(n)$ for some $n \in \mathbb{N}$.

Proof. Let $\mathcal{L}=\operatorname{Bool}(n)$ for some $n \in \mathbb{N}$, and let $X \subseteq[n]$. Since

$$
\operatorname{Atoms}(\operatorname{Bool}(n))=\{\{1\},\{2\}, \ldots,\{n\}\},
$$

and the join operation in $\operatorname{Bool}(n)$ is set union, we see directly that $\operatorname{Bool}(n)$ is atomic.

Conversely, let $\mathcal{L}=(L, \leq)$ be a semidistributive and atomic lattice. Suppose that $|\operatorname{Atoms}(\mathcal{L})|=n$. Since $\mathcal{L}$ is semidistributive, we conclude from Theorem 2.6 that every element of $\mathcal{L}$ has a canonical join representation, and since $\mathcal{L}$ is atomic we conclude by definition that any canonical join representation consists of atoms. It follows that $|L| \leq 2^{n}$.

Now let $X \subseteq \operatorname{Atoms}(\mathcal{L})$, and let $x=\bigvee X$. If $X=\varnothing$, then $x=\hat{0}$. If $X \neq \varnothing$, then $\Gamma(x) \neq \varnothing$. Suppose that there exists $z \in X \backslash \Gamma(x)$. For every $a \in \Gamma(x)$ we have $z \wedge a=\hat{0}$, since $a$ and $z$ are atoms. The meet-semidistributivity of $\mathcal{L}$ then implies $\hat{0}=z \wedge(\vee \Gamma(x))=z \wedge x=z$, which is a contradiction. Hence $X=\Gamma(x)$. We conclude that $\bigvee X$ is a canonical join representation for every $X \subseteq \operatorname{Atoms}(\mathcal{L})$. Since $\mathcal{L}$ is atomic we conclude $|L|=2^{n}$. (The map $x \mapsto \Gamma(x)$ is easily checked to be an isomorphism from $\mathcal{L}$ to $\operatorname{Bool}(\operatorname{Atoms}(\mathcal{L}))$.)

2.4. Möbius Function and Crosscuts. Let $\mathcal{P}=(P, \leq)$ be a finite poset. The Möbius function of $\mathcal{P}$ is the function $\mu_{\mathcal{P}}: P \times P \rightarrow \mathbb{Z}$ defined recursively by

$$
\mu_{\mathcal{P}}(x, y) \stackrel{\text { def }}{=} \begin{cases}1, & \text { if } x=y, \\ -\sum_{x \leq z<y} \mu_{\mathcal{P}}(x, z), & \text { if } x<y, \\ 0 & \text { otherwise. }\end{cases}
$$

An antichain of $\mathcal{P}$ is a subset of $P$ consisting of pairwise incomparable elements. A chain of $\mathcal{P}$ is a totally ordered subset of $P$. A chain is maximal if it is maximal under inclusion.

There is a nice combinatorial way to compute the Möbius function in a finite lattice $\mathcal{L}=(L, \leq)$. A crosscut of $\mathcal{L}$ is an antichain $C \subseteq P$ which contains neither 0 nor $\hat{1}$ and such that any maximal chain of $\mathcal{L}$ intersects $C$ exactly once. Examples for crosscuts are the sets of atoms or coatoms. A subset $X \subseteq L$ is spanning if $\wedge X=\hat{0}$ and $\bigvee X=\hat{1}$. The following result is known as the Crosscut Theorem.

Theorem 2.11 ([27, Theorem 3]). Let $\mathcal{L}=(L, \leq)$ be a finite lattice and let $C \subseteq L$ be a crosscut. We have

$$
\mu_{\mathcal{L}}(\hat{0}, \hat{1})=\sum_{X \subseteq C \text { spanning }}(-1)^{|X|} .
$$

In the lattice in Figure $1 a$, the set $C=\left\{a_{1}, a_{2}, a_{3}\right\}$ is a crosscut, and the spanning subsets of $C$ are $C$ itself and every subset of $C$ of size 2 . We conclude that $\mu(\hat{0}, \hat{1})=$ 2 , which can also be verified by hand.

We obtain the following result, which may also be concluded from [14, Theorems 5.1.3 and 5.4.1]. 
Theorem 2.12. If $\mathcal{L}$ is a meet-semidistributive lattice, then $\mu_{\mathcal{L}}(\hat{0}, \hat{1}) \in\{-1,0,1\}$.

Proof. This follows from Proposition 2.9 and Theorem 2.11.

Recall that the order complex of a finite poset $\mathcal{P}$ is the simplicial complex whose faces correspond to the chains of $\mathcal{P}$. If $\mathcal{P}=(P, \leq)$ is bounded (i.e. if it has a least element $\hat{0}$ and a greatest element $\hat{1})$ then we call the poset $\overline{\mathcal{P}} \stackrel{\text { def }}{=}(P \backslash\{\hat{0}, \hat{1}\}, \leq)$ the proper part of $\mathcal{P}$. A bounded poset is spherical if the order complex of its proper part is homotopy equivalent to a sphere.

A famous result of P. Hall states that $\mu_{\mathcal{P}}(\hat{0}, \hat{1})$ equals the reduced Euler characteristic of the order complex of $\overline{\mathcal{P}}$; see [29, Proposition 3.8.6]. It thus follows from Theorem 2.12 that a meet-semidistributive lattice $\mathcal{L}$ is spherical if and only if $\mu_{\mathcal{L}}(\hat{0}, \hat{1}) \neq 0$.

We have the following characterization of spherical meet-semidistributive lattices.

Proposition 2.13. In a meet-semidistributive lattice $\mathcal{L}$ we have $\bigvee \operatorname{Atoms}(\mathcal{L})=\hat{1}$ if and only if $\mu_{\mathcal{L}}(\hat{0}, \hat{1}) \neq 0$.

Proof. Let $e$ (resp. o) denote the number of spanning subsets of $\operatorname{Atoms}(\mathcal{L})$ of even (resp. odd) size. Proposition 2.9 implies that $e+o \leq 1$.

If $\mu_{\mathcal{L}}(\hat{0}, \hat{1})=0$, then Theorem 2.11 implies that $e=o$, which forces $e=o=0$. Hence $\bigvee \operatorname{Atoms}(\mathcal{L})<\hat{1}$.

Conversely if $\mu_{\mathcal{L}}(\hat{0}, \hat{1}) \neq 0$, then Theorem 2.12 implies $\mu_{\mathcal{L}}(\hat{0}, \hat{1})= \pm 1$. Hence we have either $e=1$ and $o=0$, or $e=0$ and $o=1$. Proposition 2.9 implies that $\operatorname{VAtoms}(\mathcal{L})=\hat{1}$.

\section{The Core Label Order of a Congruence-Uniform Lattice}

3.1. The Core Label Order. Let $\mathcal{L}=(L, \leq)$ be a congruence uniform lattice. N. Reading defined in [26, Section 9-7.4] an alternate partial order on $L$ as follows. The nucleus of $x \in L$ is

$$
x_{\downarrow} \stackrel{\text { def }}{=} \bigwedge_{y \in L: y<x} y .
$$

The terminology is due to the fact that the interval $\left[x_{\downarrow}, x\right]$ is a nuclear interval, i.e. an interval in which the top element is the join of all upper covers of the bottom element. Moreover, we call the interval $\left[x_{\downarrow}, x\right]$ the core of $x$. We also define

$$
\Psi_{\mathcal{L}}(x) \stackrel{\text { def }}{=}\left\{j_{\operatorname{cg}(u, v)} \mid x_{\downarrow} \leq u \lessdot v \leq x\right\} .
$$

In other words, $\Psi_{\mathcal{L}}(x)$ is the set of "labels" of the core of $x$, or simply the core label set of $x$. (Observe that we may label every cover relation $u \lessdot v$ in a congruenceuniform lattice by the join-irreducible element $j_{\operatorname{cg}(u, v)}$.) We omit the subscript $\mathcal{L}$ whenever no confusion can arise.

There is an easy way to obtain $j_{\operatorname{cg}(u, v)}$ from the cover relation $u \lessdot v$ without having to compare congruences. Recall that two cover relations $x_{1} \lessdot y_{1}$ and $x_{2} \lessdot y_{2}$ are perspective if either $y_{1} \vee x_{2}=y_{2}$ and $y_{1} \wedge x_{2}=x_{1}$ or $y_{2} \vee x_{1}=y_{1}$ and $y_{2} \wedge$ $x_{1}=x_{2}$. The next result implies that $\Psi(x)$ contains precisely those join-irreducible elements $j$ such that $\left(j_{*}, j\right)$ is perspective to some cover relation in $\left[x_{\downarrow}, x\right]$. 


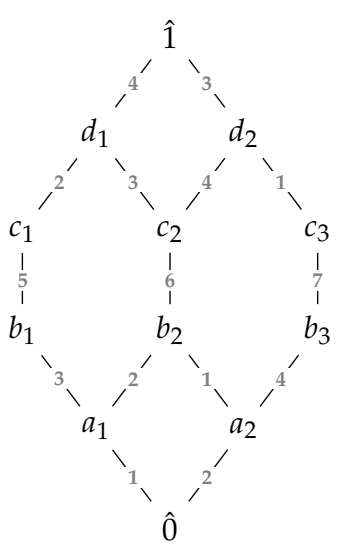

(a) A congruence-uniform lattice.

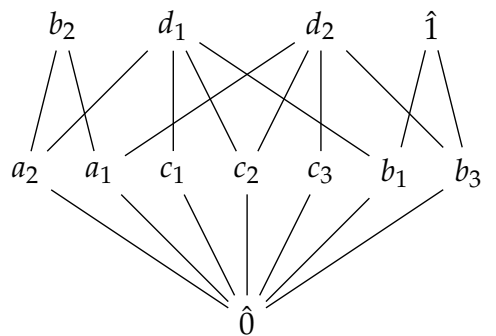

(b) The core label order of the lattice in Figure 6a.

Figure 6. A congruence-uniform lattice whose core label order is not a lattice.

Lemma 3.1 ([12, Lemma 2.6]). Let $\mathcal{L}=(L, \leq)$ be a congruence-uniform lattice, and let $u, v \in L$ such that $u \lessdot v$. For $j \in \mathcal{J}(\mathcal{L})$ holds $\operatorname{cg}(j)=\operatorname{cg}(u, v)$ if and only if $j_{*} \lessdot j$ and $u \lessdot v$ are perspective.

For $x, y \in L$ we define $x \sqsubseteq y$ if and only if $\Psi(x) \subseteq \Psi(y)$, and we call the poset

$$
\operatorname{CLO}(\mathcal{L}) \stackrel{\text { def }}{=}(L, \sqsubseteq)
$$

the core label order of $\mathcal{L}$. The assignment $x \mapsto \Psi_{\mathcal{L}}(x)$ is injective by virtue of Theorem 2.1 and the fact that the map $j \mapsto \operatorname{cg}(j)$ is a bijection. Therefore, the relation $\sqsubseteq$ is indeed a partial order.

The main motivation for this definition comes from the poset of regions in a hyperplane arrangement. We have the following result.

Theorem 3.2 ([26, Section 9-7.4]). Let $\mathcal{L}$ be a poset of regions of a hyperplane arrangement. If $\mathcal{L}$ is a congruence-uniform lattice, then $\operatorname{CLO}(\mathcal{L})$ is a lattice.

The hyperplane arrangements that have posets of regions which are congruenceuniform lattices are characterized in [26, Corollary 9-7.22]. In the case described in Theorem 3.2, the poset $C L O(\mathcal{L})$ is usually referred to as the shard intersection order. However, in the general setting, the term "shard" is not really justified, and the collection $\{\Psi(x) \mid x \in L\}$ is in general not closed under intersections.

If $\mathcal{L}$ is a congruence-uniform lattice that does not arise as a poset of regions of some hyperplane arrangement, then the core label order need not be a lattice. Consider for instance the lattice $\mathcal{L}$ in Figure 6a. The cover relations of $\mathcal{L}$ are labeled so that they reflect the linear extension

$$
a_{1} \prec a_{2} \prec b_{1} \prec b_{3} \prec c_{1} \prec c_{2} \prec c_{3}
$$

of the poset of join-irreducible elements of $\mathcal{L}$. Observe that this labeling also indicates how $\mathcal{L}$ arises from doublings by intervals: the $i^{\text {th }}$ join-irreducible element in 
this linear extension is created in the $i^{\text {th }}$ doubling step. We thus obtain

$$
\begin{array}{llll}
\Psi(\hat{0})=\varnothing, & \Psi\left(a_{1}\right)=\{1\}, & \Psi\left(a_{2}\right)=\{2\}, & \Psi\left(b_{1}\right)=\{3\}, \\
\Psi\left(b_{2}\right)=\{1,2\}, & \Psi\left(b_{3}\right)=\{4\}, & \Psi\left(c_{1}\right)=\{5\}, & \Psi\left(c_{2}\right)=\{6\}, \\
\Psi\left(c_{3}\right)=\{7\}, & \Psi\left(d_{1}\right)=\{2,3,5,6\}, & \Psi\left(d_{2}\right)=\{1,4,6,7\}, & \Psi(\hat{1})=\{3,4\} .
\end{array}
$$

Figure $6 \mathrm{~b}$ shows the core label order of $\mathcal{L}$. We observe that this poset is a meetsemilattice, i.e. any two elements have a meet, but it is not a lattice since it does not have a greatest element. We observe further that $\mathcal{L}$ is not spherical.

Let us close this section with the following result, which implies that there exists a bijection on the elements of a congruence-uniform lattice that swaps canonical join representations with canonical meet representations.

Lemma 3.3 ([12, Lemma 2.12]). Let $\mathcal{L}=(L, \leq)$ be a congruence-uniform lattice, and let $y \in L$. Let $a_{1}, a_{2}, \ldots, a_{s}$ be some elements that cover $y$, and let $x=a_{1} \vee a_{2} \vee \cdots \vee a_{\text {s. }}$. Then there exist elements $c_{1}, c_{2}, \ldots, c_{s}$ that that are covered by $x$ such that $y=c_{1} \wedge c_{2} \wedge$ $\cdots \wedge c_{s}$ and $\operatorname{cg}\left(y, a_{i}\right)=\operatorname{cg}\left(c_{i}, x\right)$ for all $i \in[s]$.

In particular, if $x$ and $y$ are as in Lemma 3.3, then we have $x_{\downarrow} \leq y$.

\section{Conditions for the Lattice Property of $\mathrm{ClO}(\mathcal{L})$}

4.1. A Necessary Condition for the Lattice Property of $\operatorname{CLO}(\mathcal{L})$. Our first main result, Theorem 1.1, which we are going to prove in the remainder of this section, establishes that the core label order of $\mathcal{L}$ is a lattice only if $\mathcal{L}$ is spherical.

Lemma 4.1. If $j \in \Psi(x)$, then $j \leq x$.

Proof. By definition, $j \in \Psi(x)$ means that $j=j_{\operatorname{cg}(u, v)}$ for some $x_{\downarrow} \leq u \lessdot v \leq x$. In particular, $\operatorname{cg}(u, v)=\operatorname{cg}(j)$, so that Lemma 3.1 implies that $j \vee u=v$, and thus $j \leq v \leq x$.

Lemma 4.2. We have $x=\bigvee \Psi(x)$.

Proof. By Proposition 2.8, we have $\Gamma(x) \subseteq \Psi(x)$, and by definition follows $x=$ $\bigvee \Gamma(x)$. For $j \in \Psi(x) \backslash \Gamma(x)$ we conclude from Lemma 4.1 that $j \leq x$, and therefore $x=x \vee j$. This yields the claim.

Corollary 4.3. If $\Psi(x) \subseteq \Psi(y)$, then $x \leq y$.

Proof. This is a direct computation using Lemma 4.2.

Corollary 4.4. The greatest element of $\mathcal{L}$ is maximal in $\operatorname{CLO}(\mathcal{L})$.

Proof. Let $\hat{1}$ denote the greatest element of $\mathcal{L}$. If there is $x \in L$ with $\Psi(\hat{1}) \subseteq \Psi(x)$, then by Corollary 4.3 we conclude $\hat{1} \leq x$, which implies $x=\hat{1}$, since $\hat{1}$ is maximal in $\mathcal{L}$.

Lemma 4.5. We have $\Psi(\hat{1})=\mathcal{J}(\mathcal{L})$ if and only if $\mu_{\mathcal{L}}(\hat{0}, \hat{1}) \neq 0$.

Proof. Let $C$ denote the set of coatoms of $\mathcal{L}$.

If $\mu_{\mathcal{L}}(\hat{0}, \hat{1}) \neq 0$, then the dual of Proposition 2.13 implies that $\wedge C=\hat{0}$, so that by definition $\Psi(\hat{1})$ contains all join-irreducible elements of $\mathcal{L}$.

If $\mu_{\mathcal{L}}(\hat{0}, \hat{1})=0$, then the dual of Proposition 2.13 implies that $\wedge C=x>\hat{0}$. In particular, there is some $a \in \operatorname{Atoms}(\mathcal{L})$ with $a \leq x$. If $a \in \Psi(\hat{1})$, then there exist 


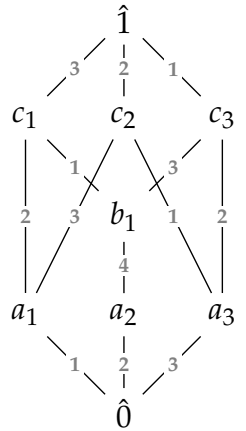

(a) A spherical congruence-uniform lattice.

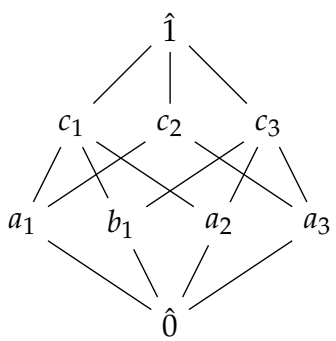

(b) The core label order of the lattice in Figure 7a.

Figure 7. A spherical congruence-uniform lattice whose core label order is not a lattice.

$u, v \in L$ with $x \leq u \lessdot v$ such that $\operatorname{cg}(u, v)=\operatorname{cg}(a)$. Lemma 3.1 implies $a \vee u=v$. However, $a \leq x \leq u$ implies $a \vee u=u$, which is a contradiction. We conclude that $a \notin \Psi(\hat{1})$.

Lemma 4.6. There exists a greatest element in $\operatorname{CLO}(\mathcal{L})$ if and only if $\mu_{\mathcal{L}}(\hat{0}, \hat{1}) \neq 0$.

Proof. Let $C$ denote the set of coatoms of $\mathcal{L}$.

If $\mu_{\mathcal{L}}(\hat{0}, \hat{1}) \neq 0$, then Lemma 4.5 implies $\Psi(\hat{1})=\mathcal{J}(\mathcal{L})$. It follows that for any $x \in L$ we have $\Psi(x) \subseteq \Psi(\hat{1})$, which implies that $\hat{1}$ is the unique maximal element of $\operatorname{CLO}(\mathcal{L})$.

If $\mu_{\mathcal{L}}(\hat{0}, \hat{1})=0$, then Lemma 4.5 implies that there is $j \in \mathcal{J}(\mathcal{L})$ with $j \notin \Psi(\hat{1})$. Since $\hat{1}$ is maximal in $\operatorname{CLO}(\mathcal{L})$ by Corollary 4.4 , we conclude that it is incomparable to $j$ in $\operatorname{CLO}(\mathcal{L})$. The maximality of $\hat{1}$ implies further that there is no upper bound for $\hat{1}$ and $j$ in $\operatorname{CLO}(\mathcal{L})$, which therefore does not have a greatest element.

We can now conclude the proof of Theorem 1.1.

Proof of Theorem 1.1. If $\mu_{\mathcal{L}}(\hat{0}, \hat{1})=0$, then Lemma 4.6 implies that $\operatorname{CLO}(\mathcal{L})$ does not have a greatest element. Since $\mathcal{L}$ is finite, $\operatorname{CLO}(\mathcal{L})$ can therefore not be a lattice.

The example in Figure 7 illustrates that there exist spherical congruence-uniform lattices whose core label order is not a lattice. The labels reflect the linear extension $a_{1} \prec a_{2} \prec a_{3} \prec b_{1}$ of the poset of join-irreducible elements of this lattice.

Observe that Figure 7a is isomorphic to Bool(3) doubled by an atom, and it is exactly this doubling that kills the lattice property of the core label order. We conclude the following result.

Proposition 4.7. Let $x, y \in L$ be such that $\Psi(j) \subseteq \Psi(x) \cap \Psi(y)$ for some $j \in \mathcal{J}(\mathcal{L})$ which satisfies $j \in\left[x_{\downarrow}, x\right] \cap\left[y_{\downarrow}, y\right]$. The core label order of $\mathcal{L}[j]$ is not a lattice.

Proof. We identify the element $(z, i) \in L[j]$ with $z$, except for the case $z=j$ and $i=1$; in this case we denote the element $(j, 1)$ by $j^{\prime}$. We conclude from Lemma 2.3 that $j^{\prime} \in \mathcal{J}(\mathcal{L}[j])$. Moreover, we have $j<j^{\prime}$ in $\mathcal{L}[j]$. 


\begin{tabular}{c||c|c|c|c|c|c|c|c|c|c|c|c|c|c}
$n$ & 1 & 2 & 3 & 4 & 5 & 6 & 7 & 8 & 9 & 10 & 11 & 12 & 13 & 14 \\
\hline \hline$l_{n}$ & 1 & 1 & 1 & 2 & 5 & 15 & 53 & 222 & 1078 & 5994 & 37622 & 262776 & 2018305 & 16873364 \\
\hline$c_{n}$ & 1 & 1 & 1 & 2 & 4 & 9 & 22 & 60 & 174 & 534 & 1720 & 5767 & 20013 & 71545 \\
\hline$S_{n}$ & 1 & 1 & 0 & 1 & 1 & 2 & 3 & 8 & 17 & 45 & 123 & 367 & 1148 & 3792 \\
\hline$S_{n}$ & 1 & 1 & 0 & 1 & 1 & 2 & 3 & 8 & 16 & 41 & 107 & 304 & 891 & 2735
\end{tabular}

Table 1. Numerology of congruence-uniform lattices.

Corollary 4.3 implies that $j$ is a lower bound for $x$ and $y$ in $\mathcal{L}$, and it follows by construction that $j^{\prime}$ is a lower bound for $x$ and $y$ in $\mathcal{L}[j]$. We thus have $\Psi_{\mathcal{L}[j]}(x)=$ $\Psi_{\mathcal{L}}(x) \cup\left\{j^{\prime}\right\}$ and $\Psi_{\mathcal{L}[j]}(y)=\Psi_{\mathcal{L}}(y) \cup\left\{j^{\prime}\right\}$. By assumption we have $\{j\}=\Psi_{\mathcal{L}[j]}(j) \subseteq$ $\Psi_{\mathcal{L}[j]}(x) \cap \Psi_{\mathcal{L}[j]}(y)$ and by construction follows $\left\{j^{\prime}\right\}=\Psi_{\mathcal{L}[j]}\left(j^{\prime}\right) \subseteq \Psi_{\mathcal{L}[j]}(x) \cap$ $\Psi_{\mathcal{L}[j]}(y)$. We conclude that $\mathrm{CLO}(\mathcal{L})$ is not a lattice.

We certainly cannot leave out the extra condition on $j$ in Proposition 4.7, since we need to double at an interval contained in $\left[x_{\downarrow}, x\right] \cap\left[y_{\downarrow}, y\right]$ in order to ensure that $\Psi_{\mathcal{L}}(x) \neq \Psi_{\mathcal{L}[j]}(x)$ and $\Psi_{\mathcal{L}}(y) \neq \Psi_{\mathcal{L}[j]}(y)$. We may now prove Theorem 1.2.

Proof of Theorem 1.2. Let $\mathcal{L}$ be a congruence-uniform lattice with at least three atoms $a, b, c$. Let $x=a \vee b$ and $y=b \vee c$. Since $\mathcal{L}$ is join semidistributive by Proposition 2.5, we conclude that $x \neq y$ as well as $\Gamma(x)=\{a, b\}$ and $\Gamma(y)=\{b, c\}$.

Proposition 2.8 implies that there are exactly two lower covers of $x$, say $r_{1}$ and $r_{2}$, and let $r=r_{1} \wedge r_{2}$. Since $r<x=a \vee b$, we conclude that $a \not \leq r$. (Analogously follows $b \not \leq r$.) Since $a$ and $b$ are atoms we conclude that $a \wedge r=\hat{0}=b \wedge r$. The meet-semidistributivity of $\mathcal{L}$ implies that $\hat{0}=(a \vee b) \wedge r=x \wedge r=r$. We conclude that $\Psi(b) \subseteq \Psi(x)$. By symmetry we obtain $\Psi(b) \subseteq \Psi(y)$.

Since we have just seen that $x_{\downarrow}=\hat{0}=y_{\downarrow}$, and since $b \leq x$ and $b \leq y$ by construction, we conclude that $b \in[\hat{0}, x] \cap[\hat{0}, y]$. We can therefore apply Proposition 4.7, which proves that $\operatorname{CLO}(\mathcal{L}[b])$ is not a lattice. Moreover, we have $\mu_{\mathcal{L}[b]}(\hat{0}, \hat{1})=$ $\mu_{\mathcal{L}}(\hat{0}, \hat{1})$, so that $\mathcal{L}[b]$ is spherical if and only if $\mathcal{L}$ is.

The smallest congruence-uniform lattice with three atoms is Bool(3), which implies that the smallest example of the previously described construction has nine elements.

It can be quickly verified that sphericity of $\mathcal{L}$ is a sufficient condition for the lattice property of $\operatorname{CLO}(\mathcal{L})$ if $\mathcal{L}$ has at most eight elements. The example in Figure 7 is thus the smallest spherical congruence-uniform lattice whose core label order is not a lattice. Table 1 lists the number of congruence-uniform lattices of size $\leq 14$, and the number of such lattices that are spherical and have an core label order that is a lattice. These numbers were obtained with the help of Sage-Combinat [30,31]. Let us use the following abbreviations:

- $l_{n}$ denotes the number of all lattices of size $n$; see [28, A006966],

- $c_{n}$ denotes the number of all congruence-uniform lattices of size $n$; see [28, A292790],

- $s_{n}$ denotes the number of spherical congruence-uniform lattices of size $n$; see [28, A292852], and

- $S_{n}$ denotes the number of all congruence-uniform lattices of size $n$ whose core label order is a lattice; see [28, A292853]. 
4.2. The Intersection Property. Let $\mathcal{L}=(L, \leq)$ be a congruence-uniform lattice. We say that $\mathcal{L}$ has the intersection property if for all $x, y \in L$ there exists some $z \in L$ with $\Psi(x) \cap \Psi(y)=\Psi(z)$. It turns out that $\mathcal{L}$ having the intersection property is equivalent to $\operatorname{CLO}(\mathcal{L})$ being a meet-semilattice.

Theorem 4.8. The core label order of a congruence-uniform lattice $\mathcal{L}$ is a meet-semilattice if and only if $\mathcal{L}$ has the intersection property.

Proof. If $\mathcal{L}=(L, \leq)$ has the intersection property, then $\{\Psi(x) \mid x \in L\}$ is closed under intersections, which means that $\operatorname{CLO}(\mathcal{L})$ is a meet-semilattice.

Conversely, suppose that $\mathrm{CLO}(\mathcal{L})$ is a meet-semilattice, and let $x, y \in L$. By assumption the meet $z$ of $x$ and $y$ in $\operatorname{CLO}(\mathcal{L})$ exists, and by construction we have $\Psi(z) \subseteq \Psi(x) \cap \Psi(y)$. On the other hand, however, if $j \in \Psi(x) \cap \Psi(y)$, then $j \in$ $\mathcal{J}(\mathcal{L})$ and we have $\Psi(j)=\{j\}$. It follows then that $j \sqsubseteq x$ and $j \sqsubseteq y$, and therefore $j \sqsubseteq z$. This implies $j \subseteq \Psi(z)$, from which follows that $\Psi(z)=\Psi(x) \cap \Psi(y)$. Consequently, $\mathcal{L}$ has the intersection property.

The proof of the implication " $\mathrm{CLO}(\mathcal{L})$ is a meet-semilattice implies $\mathcal{L}$ has the intersection property" was suggested to us by a referee. The proof of Theorem 1.3 is now immediate.

Proof of Theorem 1.3. The first part is precisely Theorem 4.8. Lemma 4.6 states that $\operatorname{CLO}(\mathcal{L})$ has a greatest element if and only if $\mathcal{L}$ is spherical. It is a classical latticetheoretic result that a finite meet-semilattice with a greatest element is a lattice; see for instance [26, Lemma 9-2.1].

Of course, we have just shifted the question when $\operatorname{CLO}(\mathcal{L})$ is a meet-semilattice to the question when $\mathcal{L}$ has the intersection property.

It follows from [26, Section 9-7.4] that a congruence-uniform lattice of regions has the intersection property.

We now prove that the intersection property is inherited to quotient lattices.

Lemma 4.9. Let $\mathcal{L}=(L, \leq)$ be a congruence-uniform lattice and let $\Theta \in \operatorname{Con}(\mathcal{L})$. Let $\Sigma=\left\{j \in \mathcal{J}(\mathcal{L}) \mid\left(j_{*}, j\right) \in \Theta\right\}$. For $x \in L$ the set $\Psi_{\mathcal{L} / \Theta}\left([x]_{\Theta}\right)$ is in bijection with $\Psi_{\mathcal{L}}(x) \backslash \Sigma$.

Proof. The map

$$
f: \mathcal{J}(\mathcal{L}) \backslash \Sigma \rightarrow \mathcal{J}(\mathcal{L} / \Theta), \quad j \mapsto[j]_{\Theta}
$$

is by construction a bijection. Let $x \in L$. If $j \in \Psi_{\mathcal{L}}(x)$, then we have $j=j_{\operatorname{cg}(u, v)}$ for some $x_{\downarrow} \leq u \lessdot v \leq x$.

If $j \in \Sigma$, then we have $(u, v) \in \Theta$, and therefore $[u]_{\Theta}=[v]_{\Theta}$. In particular we conclude $[j]_{\Theta} \notin \Psi_{\mathcal{L} / \Theta}\left([x]_{\Theta}\right)$. If $j \notin \Sigma$, then we have $[u]_{\Theta} \lessdot[v]_{\Theta}$, and we conclude $[j]_{\Theta} \in \Psi_{\mathcal{L} / \Theta}\left([x]_{\Theta}\right)$.

It follows that $f$ is the desired bijection from $\Psi_{\mathcal{L}}(x) \backslash \Sigma$ to $\Psi_{\mathcal{L} / \Theta}\left([x]_{\Theta}\right)$.

Proposition 4.10. Let $\mathcal{L}$ be a spherical congruence-uniform lattice. For every $\Theta \in$ $\operatorname{Con}(\mathcal{L})$ the quotient lattice $\mathcal{L} / \Theta$ is spherical, too.

Proof. Let $\Sigma=\left\{j \in \mathcal{J}(\mathcal{L}) \mid\left(j_{*}, j\right) \in \Theta\right\}$, and let $A=\operatorname{Atoms}(\mathcal{L}) \cap \Sigma$, and let $B=\operatorname{Atoms}(\mathcal{L}) \backslash A$. Then we have

$$
\operatorname{Atoms}(\mathcal{L} / \Theta)=\left\{[b]_{\Theta} \mid b \in B\right\} .
$$


Let $x=\bigvee B$; the element $[x]_{\Theta}=\bigvee_{b \in B}[b]_{\Theta}$ is thus the join of all atoms in $\mathcal{L} / \Theta$. Moreover, since $\mathcal{L}$ is spherical, Proposition 2.13 implies that $\hat{1}=(\bigvee A) \vee(\vee B)$. For $a \in A$, we have by definition that $[a \vee x]_{\Theta}=[\hat{0} \vee x]_{\Theta}=[x]_{\Theta}$. We thus obtain

$$
[x]_{\Theta}=[(\bigvee A) \vee x]_{\Theta}=[(\bigvee A) \vee(\bigvee B)]_{\Theta}=[\hat{1}]_{\Theta} .
$$

Proposition 2.13 thus implies that $\mathcal{L} / \Theta$ is spherical.

Proposition 4.11. Let $\mathcal{L}$ be a congruence-uniform lattice with the intersection property. For every $\Theta \in \operatorname{Con}(\mathcal{L})$ the quotient lattice $\mathcal{L} / \Theta$ has the intersection property, too.

Proof. Let $\Sigma=\left\{j \in \mathcal{J}(\mathcal{L}) \mid\left(j_{*}, j\right) \in \Theta\right\}$, and let $f$ be the bijection from Lemma 4.9. Consequently, for $x \in L$ we have $\Psi_{\mathcal{L} / \Theta}\left([x]_{\Theta}\right)=f\left(\Psi_{\mathcal{L}}(x) \backslash \Sigma\right)$.

Fix $x, y \in L$. Since $\mathcal{L}$ has the intersection property we can find $z \in L$ with $\Psi_{\mathcal{L}}(z)=\Psi_{\mathcal{L}}(x) \cap \Psi_{\mathcal{L}}(y)$. We then have

$$
\begin{aligned}
& \Psi_{\mathcal{L} / \Theta}\left([x]_{\Theta}\right) \cap \Psi_{\mathcal{L} / \Theta}\left([y]_{\Theta}\right)=f\left(\Psi_{\mathcal{L}}(x) \backslash \Sigma\right) \cap f\left(\Psi_{\mathcal{L}}(y) \backslash \Sigma\right)= \\
& f\left(\left(\Psi_{\mathcal{L}}(x) \cap \Psi_{\mathcal{L}}(y)\right) \backslash \Sigma\right)=f\left(\Psi_{\mathcal{L}}(z) \backslash \Sigma\right)=\Psi_{\mathcal{L} / \Theta}\left([z]_{\Theta}\right) .
\end{aligned}
$$

It follows that $\mathcal{L} / \Theta$ has the intersection property.

We conclude this section with the proof of Theorem 1.4.

Proof of Theorem 1.4. It follows from Proposition 2.2 that $\mathcal{L} / \Theta$ is congruence-uniform, and Propositions 4.10 and 4.11 imply that $\mathcal{L} / \Theta$ is spherical and has the intersection property. The claim then follows from Theorem 1.3.

\section{A NeW Characterization of Boolean Lattices}

In this section we attempt to give a conceptual interpretation of the set of core labels of some element $x$ of a congruence-uniform lattice. Proposition 2.8 implies that $\Gamma(x) \subseteq \Psi(x)$ holds for all $x \in L$. We show now that equality holds precisely in the case where $\left[x_{\downarrow}, x\right]$ is a Boolean lattice.

Proposition 5.1. Let $\mathcal{L}=(L, \leq)$ be a congruence-uniform lattice, and let $x \in L$. We have $\Gamma(x)=\Psi(x)$ if and only if $\left[x_{\downarrow}, x\right] \cong \operatorname{Bool}(k)$, where $k=|\Gamma(x)|$.

Proof. Proposition 2.2 implies that intervals of congruence-uniform lattices are congruence uniform again. We may thus assume that $x=\hat{1}$ and $x_{\downarrow}=\hat{0}$. Moreover, let $k=|\operatorname{Atoms}(\mathcal{L})|$, which in view of Lemma 3.3 means that $|\Gamma(\hat{1})|=k$.

By construction we find that $\Gamma(\hat{1})=\Psi(\hat{1})$ if and only if $\mathcal{J}(\mathcal{L})=\operatorname{Atoms}(\mathcal{L})$, which is equivalent to $\mathcal{L}$ being atomic. The claim follows now from Theorem 2.10.

Consequently, the size of the set $\Psi(x) \backslash \Gamma(x)$ tells us "how far off" the interval $\left[x_{\downarrow}, x\right]$ is from a Boolean lattice. In other words, $|\Psi(x) \backslash \Gamma(x)|$ is precisely the number of doublings that we need to "undo" in order to turn $\left[x_{\downarrow}, x\right]$ into a Boolean lattice. For a congruence-uniform lattice $\mathcal{L}=(L, \leq)$ we may thus define the Boolean defect of $\mathcal{L}$ by

$$
\operatorname{bdef}(\mathcal{L}) \stackrel{\text { def }}{=} \sum_{x \in L}|\Psi(x) \backslash \Gamma(x)| .
$$

The lattice in Figure 6a has Boolean defect 4, and the lattice in Figure 7a has Boolean defect 3 . 
Proposition 5.2. Let $\mathcal{L}=(L, \leq)$ be a congruence-uniform lattice. We have bdef $(\mathcal{L})=$ 0 if and only if $\left[x_{\downarrow}, x\right]$ is isomorphic to a Boolean lattice for all $x \in L$.

Proof. This follows from Proposition 5.1.

Corollary 5.3. A spherical congruence-uniform lattice $\mathcal{L}$ has $\operatorname{bdef}(\mathcal{L})=0$ if and only if $\mathcal{L} \cong \operatorname{Bool}(n)$ for some $n \in \mathbb{N}$.

Proof. This follows from the dual of Proposition 2.13 and Proposition 5.2.

Perhaps the simplest example of a non-spherical congruence-uniform lattice with Boolean defect 0 is a chain of length at least three.

If $\mathcal{L}$ is join semidistributive, then for every $X \subseteq \operatorname{Atoms}(\mathcal{L})$ there exists an element $x \in L$ with $\Gamma(x)=X$. Let us define the Boolean nexus of $\mathcal{L}$ by

$$
\operatorname{Nexus}(\mathcal{L}) \stackrel{\text { def }}{=}\{x \in L \mid \Gamma(x) \subseteq \operatorname{Atoms}(\mathcal{L})\} .
$$

Proposition 5.4. Let $\mathcal{L}=(L, \leq)$ be a congruence-uniform lattice with $n$ atoms. Then $(\operatorname{Nexus}(\mathcal{L}), \leq) \cong \operatorname{Bool}(n)$.

Proof. This follows from Lemma 3.3.

Corollary 5.5. For $x \in \operatorname{Nexus}(\mathcal{L})$ we have $\Psi(x)=\{j \in \mathcal{J}(\mathcal{L}) \mid j \leq x\}$.

Proof. Since $\Gamma(x) \subseteq \operatorname{Atoms}(\mathcal{L})$, Lemma 3.3 implies that $x_{\downarrow}=\hat{0}$, and thus $\Psi(x)=$ $\{j \in \mathcal{J}(\mathcal{L}) \mid j \leq x\}$.

Proposition 5.6. If $\mathcal{L}=(L, \leq)$ is a congruence-uniform lattice, then the poset $(\operatorname{Nexus}(\mathcal{L}), \leq$ ) is an induced subposet of $\operatorname{CLO}(\mathcal{L})$.

Proof. We need to show that for $x, y \in \operatorname{Nexus}(\mathcal{L})$ we have $x \leq y$ if and only if $\Psi(x) \subseteq \Psi(y)$. Corollary 4.3 establishes one direction, and Corollary 5.5 implies the other.

Let $\mathcal{L}=(L, \leq)$ be a finite lattice, and let $C \subseteq L$ be a crosscut. Recall that the crosscut complex of $\mathcal{L}$ (with respect to $C$ ) is the simplicial complex $\operatorname{Cross}(\mathcal{L} ; C)$ whose ground set is $C$ and whose faces are the subsets $B \subseteq C$ for which $\bigvee B$ or $\wedge B$ exists and belongs to $L \backslash\{\hat{0}, \hat{1}\}$. (In particular, any subset of $C$ whose join is $\hat{1}$ and whose meet is 0 is not a face of $\operatorname{Cross}(\mathcal{L} ; C)$.)

If $\mathcal{L}$ is congruence uniform and we choose $C=\operatorname{Atoms}(\mathcal{L})$, then the faces of $\operatorname{Cross}(\mathcal{L} ; \operatorname{Atoms}(\mathcal{L}))$ correspond to the elements of either $\operatorname{Nexus}(\mathcal{L}) \backslash\{\hat{0}, \hat{1}\}$ (if $\mathcal{L}$ is spherical) or $\operatorname{Nexus}(\mathcal{L}) \backslash\{\hat{0}\}$ (if $\mathcal{L}$ is not spherical) via the map $X \mapsto \bigvee X$. In particular, if $|\operatorname{Atoms}(\mathcal{L})|=n$, then $\operatorname{Cross}(\mathcal{L} ; \operatorname{Atoms}(\mathcal{L}))$ is homotopy equvialent to the boundary of a $(n-1)$-simplex (if $\mathcal{L}$ is spherical) or to an $(n-1)$-simplex (if $\mathcal{L}$ is not spherical). See also [15, Section 2].

We conclude this section with the proof of Theorem 1.5, which states that the Boolean lattices are the only congruence-uniform lattices isomorphic to their core label order. This property may therefore be taken as a new characterization of Boolean lattices.

Proof of Theorem 1.5. Let $\mathcal{L} \cong \operatorname{Bool}(n)$ for some $n \in \mathbb{N}$. Proposition 5.1 implies that $\Psi(X)=\Gamma(X)$ for every $X \subseteq[n]$. For $X \subseteq[n]$ we have $X=\bigcup \Gamma(X)$, which yields $\operatorname{Bool}(n) \cong \mathrm{CLO}(\operatorname{Bool}(n))$. 


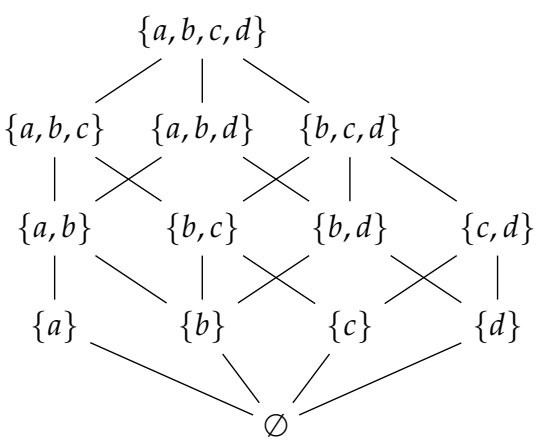

Figure 8. A lattice of closed sets.

Conversely, let $\mathcal{L}$ be such that $\mathcal{L} \cong \operatorname{CLO}(\mathcal{L})$. Suppose that $\mathcal{L}$ has $n$ atoms. We conclude that $\operatorname{CLO}(\mathcal{L})$ has $n$ atoms as well, and it is immediate from the definition that the atoms of $\operatorname{CLO}(\mathcal{L})$ are precisely the join-irreducible elements of $\mathcal{L}$. We conclude that an element of $\mathcal{L}$ is join irreducible if and only if it is an atom, which means precisely that $\mathcal{L}$ is atomic. Since $\mathcal{L}$ is also semidistributive by Proposition 2.5 we conclude from Theorem 2.10 that $\mathcal{L} \cong \operatorname{Bool}(n)$.

\section{LATTICES OF BiCLOSED SETS}

Let us finally explain what biclosed sets are. Let $S$ be a (finite) set, and let $\wp(S)$ denote the power set of $S$. A closure operator is a map cl: $\wp(S) \rightarrow \wp(S)$ which is extensive, monotone, and idempotent, i.e. which has the following three properties:

- for $X \subseteq S$ we have $X \subseteq \operatorname{cl}(X)$;

- for $X, Y \subseteq S$ we have that $X \subseteq Y$ implies $\operatorname{cl}(X) \subseteq \operatorname{cl}(Y)$; and

- for $X \subseteq S$ we have $\operatorname{cl}(\operatorname{cl}(X))=\operatorname{cl}(X)$.

A set $X \subseteq S$ is closed if $\mathrm{cl}(X)=X$. It is straightforward to verify that the family of closed sets with respect to $\mathrm{cl}$ is closed under intersection. Therefore, the poset $(\{\mathrm{cl}(X) \mid X \subseteq S\}, \subseteq)$ is in fact a lattice.

We say that $X \subseteq S$ is biclosed if both $X$ and $S \backslash X$ are closed. Let $\operatorname{Bic}(S)$ denote the set of biclosed sets of $S$ with respect to cl. We are mainly interested in the cases where $(\operatorname{Bic}(S), \subseteq)$ is a congruence-uniform lattice. The following example exhibits a spherical lattice of biclosed sets, whose core label order is not a lattice.

Example 6.1. Let $S=\{a, b, c, d\}$, and consider the closure operator given by the nontrivial assignments

$$
\operatorname{cl}(\{a, c\})=\{a, b, c\}, \quad \operatorname{cl}(\{a, d\})=\{a, b, d\}, \quad \operatorname{cl}(\{a, c, d\})=\{a, b, c, d\} .
$$

For all other $X \subseteq S$ we have $\operatorname{cl}(X)=X$. The lattice of closed sets of $\mathrm{cl}$ is shown in Figure 8. It is quickly verified that this lattice is not meet semidistributive, and thus not congruence uniform. (For instance, we have $\{b\} \wedge\{a\}=\varnothing=\{b\} \wedge\{c\}$, but $\{b\} \wedge(\{a\} \vee\{c\})=\{b\}$.) Its subposet of biclosed sets is shown in Figure 9 a, and we can verify that it is indeed a spherical congruence-uniform lattice. (It is isomorphic to the lattice in Figure 7a doubled by the coatom $c_{2}$.) The corresponding core label order, which is not a lattice, is shown in Figure 9b. 


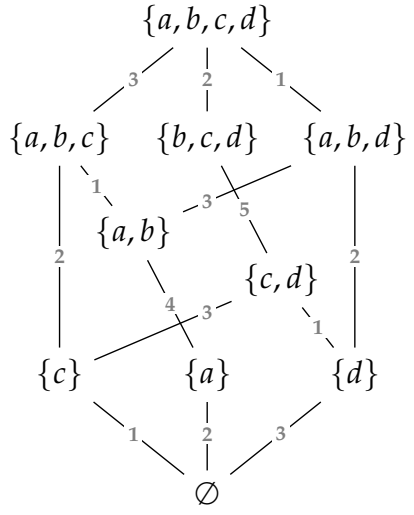

(a) A spherical concruence-uniform lattice of biclosed sets.

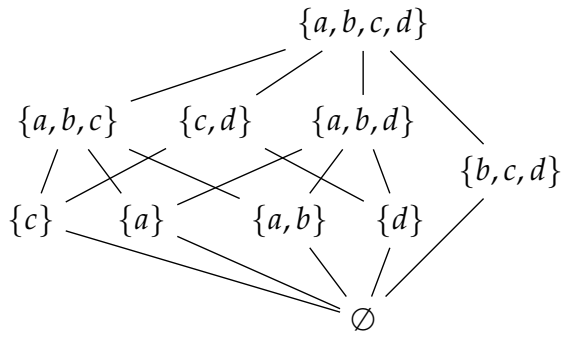

(b) The core label order of the lattice in Figure 9a.

Figure 9. The poset of biclosed sets of the lattice in Figure 8 is a spherical congruence-uniform lattice. The corresponding core label order is not a lattice.

Following T. McConville in [14, Section 2.5.1] we say that $\mathcal{X} \subseteq \wp(S)$ is ordered by single-step inclusion if for all $X, Y \in \mathcal{X}$ with $X \subsetneq Y$ there exists $x \in Y \backslash X$ such that $X \cup\{x\} \in \mathcal{X}$. We quickly observe that the set $\operatorname{Bic}(S)$ from Example 6.1 is not ordered by single-step inclusion. (For instance, this lattice has the cover relation $\{c\} \lessdot\{a, b, c\}$.) We are not aware of a spherical congruence-uniform lattice of biclosed sets which is ordered by single-step inclusion and whose core label order is not a lattice.

Problem 6.2. Find a spherical congruence-uniform lattice of biclosed sets which is ordered by single-step inclusion and whose core label order is not a lattice.

Acknowledgments. I thank Al Garver, Thomas McConville and Nathan Reading for interesting discussions on the topic, in particular for suggesting the name "core label order". Moreover, I would like to express my gratitude to an anonymous referee for many valuable suggestions that improved both content and exposition of this paper.

\section{REFERENCES}

[1] Erin Bancroft, Shard Intersections and Cambrian Congruence Classes in Type A, Dissertation, North Caroline State University, 2011.

[2] Erin Bancroft, The Shard Intersection Order on Permutations (2011), available at arXiv:1103.1910.

[3] Emily Barnard, The Canonical Join Complex (2016), available at arXiv:1610.05137.

[4] Anders Björner, Paul H. Edelman, and Günther M. Ziegler, Hyperplane Arrangements with a Lattice of Regions, Discrete \& Computational Geometry 5 (1990), 263-288.

[5] Alexander Clifton, Peter Dillery, and Alexander Garver, The Canonical Join Complex for Biclosed Sets, Algebra Universalis 79 (2018).

[6] Alan Day, Characterizations of Finite Lattices that are Bounded-Homomorphic Images or Sublattices of Free Lattices, Canadian Journal of Mathematics 31 (1979), 69-78.

[7] Alan Day, Doubling Constructions in Lattice Theory, Canadian Journal of Mathematics 44 (1992), 252-269.

[8] Paul H. Edelman, A Partial Order on the Regions of $\mathbb{R}^{n}$ Dissected by Hyperplanes, Transactions of the American Mathematical Society 283 (1984), 617-631. 
[9] Ralph Freese, Jaroslav Ježek, and James B. Nation, Free Lattices, American Mathematical Society, Providence, 1995.

[10] Nenosuke Funayama and Tadasi Nakayama, On the Distributivity of a Lattice of Lattice Congruences, Proceedings of the Imperial Academy of Tokyo 18 (1942), 553-554.

[11] Alexander Garver and Thomas McConville, Enumerative Properties of Grid-Associahedra (2017), available at arXiv: 1705.04901.

[12] Alexander Garver and Thomas McConville, Oriented Flip Graphs of Polygonal Subdivisions and Noncrossing Tree Partitions, Journal of Combinatorial Theory (Series A) 158 (2018), 126-175.

[13] George Grätzer, Lattice Theory: Foundation, Springer, Basel, 2011.

[14] Thomas McConville, Biclosed Sets in Combinatorics, Dissertation, University of Minnesota, 2015.

[15] Thomas McConville, Crosscut-Simplicial Lattices, Order 34 (2017), 465-477.

[16] Thomas McConville, Lattice Structure of Grid-Tamari Orders, Journal of Combinatorial Theory (Series A) 148 (2017), 27-56.

[17] Ralph McKenzie, Equational Bases and Nonmodular Lattice Varieties, Transactions of the American Mathematical Society 174 (1972), 1-43.

[18] James B. Nation, Unbounded Semidistributive Lattices, Algebra and Logic 39 (2000), 87-92.

[19] T. Kyle Petersen, On the Shard Intersection Order of a Coxeter Group, SIAM Journal on Discrete Mathematics 27 (2013), 1880-1912.

[20] Nathan Reading, Lattice and Order Properties of the Poset of Regions in a Hyperplane Arrangement, Algebra Universalis 50 (2003), 179-205.

[21] Nathan Reading, The Order Dimension of the Poset of Regions in a Hyperplane Arrangement, Journal of Combinatorial Theory (Series A) 104 (2003), 265-285.

[22] Nathan Reading, Clusters, Coxeter-Sortable Elements and Noncrossing Partitions, Transactions of the American Mathematical Society 359 (2007), 5931-5958.

[23] Nathan Reading, Sortable Elements and Cambrian Lattices, Algebra Universalis 56 (2007), 411-437.

[24] Nathan Reading, Noncrossing Partitions and the Shard Intersection Order, Journal of Algebraic Combinatorics 33 (2011), 483-530.

[25] Nathan Reading, Noncrossing Arc Diagrams and Canonical Join Representations, SIAM Journal on Discrete Mathematics 29 (2015), 736-750.

[26] Nathan Reading, Lattice Theory of the Poset of Regions, Lattice Theory: Selected Topics and Applications, 2016, pp. 399-487.

[27] Gian-Carlo Rota, On the Foundations of Combinatorial Theory I: Theory of Möbius Functions, Zeitschrift für Wahrscheinlichkeitstheorie und verwandte Gebiete 2 (1964), 340-368.

[28] Neil J. A. Sloane, The Online Encyclopedia of Integer Sequences. http://www . oeis . org.

[29] Richard P. Stanley, Enumerative Combinatorics, Vol. 2, Cambridge University Press, Cambridge, 2001.

[30] The Sage-Combinat Community, sage-combinat: Enhancing Sage as a Toolbox for Computer Exploration in Algebraic Combinatorics, 2018. http: // combinat. sagemath. org.

[31] The Sage Developers, Sage Mathematics Software System (Version 8.5), 2018. http://www. sagemath.org.

Institut FÜr Algebra, Technische Universität Dresden, Zellescher Weg 12-14, 01069 DRESDEN, GERMANY.

E-mail address: henri.muehle@tu-dresden.de 